\title{
Herpes simplex virus type 1-mediated transfer of neurotrophin-3 stimulates survival of chicken auditory sensory neurons
}

\author{
Estela Carnicero ${ }^{a}$, Marlies Knipper ${ }^{\mathrm{b}}$, Justin $\operatorname{Tan}^{\mathrm{b}}$, \\ Maria Teresa Alonso ${ }^{a, c}$, Thomas Schimmang ${ }^{a, c, *}$ \\ ${ }^{a}$ Instituto de Biología y Genética Molecular, Universidad de Valladolid y Consejo Superior de Investigaciones Cientificas, \\ Departamento de Bioquímica, Biología Molecular y Fisiología, Facultad de Medicina, 47005 Valladolid, Spain \\ ${ }^{b}$ Department of Otorhinolarnyngology, University of Tübingen, 72076 Tübingen, Germany \\ ${ }^{c}$ Center for Molecular Neurobiology Hamburg, University of Hamburg, Falkenried 94, 20251 Hamburg, Germany
}

Received 5 July 2001; received in revised form 12 November 2001; accepted 21 November 2001

\begin{abstract}
Neurotrophin-3 (NT-3) is one of the most potent stimulators for survival of auditory sensory neurons. Viral transfer of neurotrophins into auditory neurons may offer a route to provide a permanent supply of the growth factor and guarantee their long-term survival. Herpes simplex virus type 1 (HSV-1)-based vectors have demonstrated their effectiveness to transfer genes into peripheral sensory neurons. In the present report, we have produced a HSV-1-based amplicon vector expressing NT-3. This vector efficiently infects isolated auditory neurons and stimulates their survival during distinct developmental stages of the inner ear. Therefore, this vector may present a unique entry point to develop therapies preventing or treating hearing impairment caused by the degeneration of auditory neurons. (c) 2001 Published by Elsevier Science Ireland Ltd.
\end{abstract}

Keywords: Cochlea; Gene transfer; Chicken; Ototoxicity

Members of the neurotrophin gene family have been shown to be essential for survival of inner ear sensory neurons and maintenance of innervation with their target sensory epithelia [5]. Both brain-derived neurotrophic factor (BDNF) and neurotrophin-3 (NT-3) are expressed in the inner ear sensory epithelia of avians and mammals during development $[11,12,18]$. Simultaneously, their highaffinity receptors TrkB and $\mathrm{TrkC}$, respectively, are expressed in the peripheral auditory sensory neurons, which connect the sensory epithelia with their corresponding central auditory nuclei [10-12]. The survival of auditory sensory neurons isolated during development or adulthood is stimulated by exposure to BDNF or NT-3 $[1,7,10,11,15]$. The essential character of these neurotrophins for auditory neuron survival in vivo has been confirmed in mice lacking these factors or the corresponding Trk receptors. Mice lacking NT-3 lose more than $80 \%$ of their cochlear neurons,

* Corresponding author. Tel.: +49-40-42803-6273; fax: +49-4042803-6598.

E-mail address: schimman@epos.zmnh.uni-hamburg.de (T. Schimmang). which fail to maintain innervation with their target organs [5].

Damage to the inner ear may be caused by aging, injury, ototoxic drugs, acoustic trauma or genetic diseases [6]. The majority of hearing loss is caused by damage or loss of sensory hair cells or auditory neurons. Cochlear implants may substitute hair cell function by electrical stimulation of auditory neurons. However, survival of a critical number of neurons is required for proper function of the prosthesis. To maintain survival of auditory neurons inside the damaged inner ear, BDNF and NT-3 have been introduced via osmotic pumps $[3,4,13,16]$. Alternatively, viruses may offer a strategy to provide long-term expression of neurotrophins inside auditory neurons and maintain their function [6]. In this context, defective Herpes simplex virus type 1 (HSV-1) vectors offer some characteristics which make them the ideal gene transfer vector for the peripheral nervous system [14]. Most importantly, HSV-1 infects neurons very efficiently and acquires a state of latency without compromising normal cellular functions.

In the present study, we have developed a HSV-1-based amplicon vector containing the NT-3 gene. Gene transfer with this vector led to the efficient expression of NT-3 in 
fibroblasts. Biological activity of NT-3 was confirmed by infection of isolated avian auditory sensory neurons, which are known to respond to this growth factor [1,10,11]. By analyzing distinct developmental timepoints, we observed stimulation of survival of these neurons comparable with exogenously added NT-3. This vector may now be used to express this neurotrophin in auditory neurons in vivo and thus offer a potential tool to prevent neurodegenerative processes inside the damaged inner ear.

NT-3 has been shown to act as one of the main regulators of cochlear neuron survival in vertebrates $[1,5,10]$. We first examined the effects of recombinant NT-3 on the survival of isolated avian auditory sensory neurons. Embryonic heads were cut at the midsagittal level and after removal of brain tissue the membranous labyrinth and the cochlear duct were isolated. Ganglia were separated and neuronal cell cultures were prepared as described in detail earlier [7]. Briefly, ganglia were dissociated after treatment with $0.05 \%$ trypsin using a fire-polished Pasteur pipette. Cells were plated at a final density of 1000-2000 cells in $0.5 \mathrm{ml}$ per well. After 2 $\mathrm{h}$, the serum concentration was reduced to $1 \%$ and recombinant NT-3 or viruses were added. After addition of different concentrations of recombinant NT-3, cultures were grown for 2 days and then processed for immunocytochemical staining with a neuron-specific tubulin as described in detail earlier [7]. Briefly, after fixation in $4 \%$ paraformaldehyde and inhibition of endogenous peroxidase, cells were blocked with $20 \%$ goat serum and incubated with the class III $\beta$-tubulin (TuJ1) antibody (Babco; diluted 1:500) overnight. Cultures were washed and incubated with biotinylated IgG antibodies (diluted 1:200), for $45 \mathrm{~min}$ at room temperature. Finally, cells were washed and immunoreactivity was detected using the avidin-biotin peroxidase complex method (Vectastain Kit, Vector Laboratories). $\mathrm{TuJ} 1$-positive neurons with neurites four times longer than cell bodies were scored as surviving neurons. This number was expressed as the percentage of the total number of neurons plated. Statistics were performed using the Student's $t$-test. Compared with control cultures, we observed a significant increase of neuronal survival, with more than $50 \%$ of the neurons surviving at 1 and $5 \mathrm{ng} / \mathrm{ml}$ of NT-3 (Fig. 1A).

Having confirmed the neurotrophic potential of NT-3 on auditory sensory neurons, we were interested to transfer this growth factor directly into auditory sensory neurons. To introduce NT-3 into neurons, we used a defective HSV-1 vector or amplicon, that has been shown to permit efficient transfer of neurotrophins into auditory neurons [7,8]. For construction of pHSVnt-3 (Fig. 1B), a fragment containing the cDNA encoding for rat NT-3 was inserted into the vector pHSVpUC, and packaging and amplification of this vector were performed using standard procedures [9]. Expression of NT-3 was confirmed by an enzyme-linked immunosorbent assay (ELISA) kit (Promega) after infection of HEK293 cells. Briefly, quantification of NT-3 was determined in 96-well microplates coated with a monoclonal

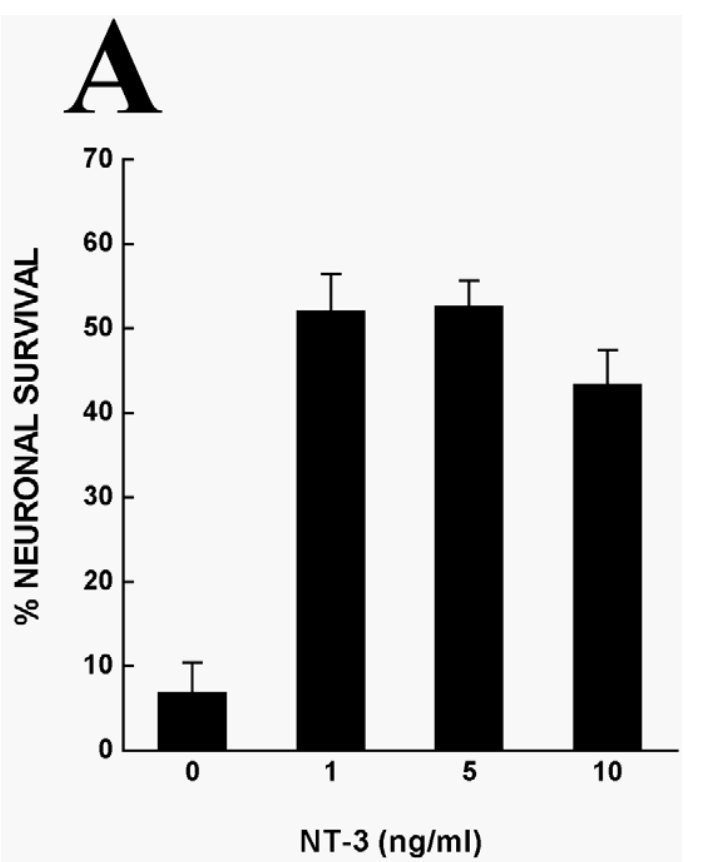

169

170

171

172

173

174

175

176
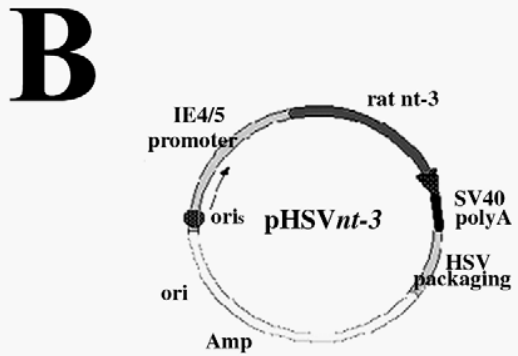

Fig. 1. Stimulation of auditory neurons by recombinant NT-3 and structure of the amplicon vector. (A) Survival of chicken auditory neurons isolated at embryonic day 8 upon addition of different amounts of recombinant NT-3. (B) Structure of the pHSVnt-3 amplicon consisting of the transcription unit composed of the IE 4/5 promoter, the rat $n t-3$ cDNA; the HSV-1 origin of replication (ori $i_{S}$ ) and the HSV-1 packaging site (' $a$ '); and sequences required for propagation in E. coli (ori and Amp).

anti-human NT-3 antibody which cross-reacts with rat NT-3. Two hundred microliters of the NT-3 standard and the supernatants from pHSVlacZ [9] expressing the Escherichia coli lacZ marker gene and pHSVnt-3-infected cells were added to each well, and the plates were incubated for $6 \mathrm{~h}$ at room temperature. After washing, $100 \mu \mathrm{l}$ of monoclonal anti-NT3 antibody, followed by $100 \mu \mathrm{l}$ of anti-mouse IgG peroxidase conjugate were added to each well and incubated for $2.5 \mathrm{~h}$. After washing, enzymatic activity was detected by adding the substrate solution for $15 \mathrm{~min}$. Absorbance values were measured at $450 \mathrm{~nm}$ using an ELISA reader (Dynatech MR5000). Supernatants from cultures infected with $1.3 \times 10^{3}$ infectious vector units (ivu) of pHSVnt-3 contained an average of $278 \mathrm{pg} / \mathrm{ml}$ of NT-3, whereas no significant amounts were obtained from cells transduced with up to $3.1 \times 10^{4}$ ivu of pHSVlacZ.

To verify biological activity of NT-3, we infected isolated avian auditory sensory neurons which have been shown to 
respond to this growth factor during distinct stages of avian development [1]. We transduced cochlear neurons cultures isolated between embryonic days 5.5 (E5.5) and E14 with $1.65-10^{4} \mathrm{ivu} / \mathrm{ml}$ of pHSVnt-3 (multiplicity of infection (MOI): 8 ) and $3.1 \times 10^{4} \mathrm{ivu} / \mathrm{ml}$ of pHSVlacZ (MOI: 15 ), respectively, and examined the effects after 2 days. HSV1-mediated transfer of NT-3 resulted in an increased survival response of cochlear neurons isolated between E5.5 and E9, compared with cultures infected with a prototype amplicon vector pHSVlacZ or uninfected cultures (Figs. 2A,B and 3). Compared with neurons grown in the presence of exogenously added NT-3, a similar amount of neurons survived in cultures transduced with pHSVnt-3 at these stages. Neuronal cultures prepared from E14 of development showed no increased survival response to pHSVnt-3 (Figs. 2C,D and 3). Similarly, exogenously added recombinant NT-3 had previously been demonstrated to have no stimulatory effect at E11 and E13 [1].

The present report underlines the neurotrophic role of NT3 during innervation of the chicken cochlea. Cochlear neurons respond to NT-3 between E5 and E9 in vitro when peripheral fibers of the cochlear ganglion invade the sensory epithelium and start early synaptogenesis in vivo. After E9, no increased survival of auditory neurons was observed upon stimulation by NT-3. These observations agree with the expression of NT-3 in vivo, which has been detected at E6 and E8, but not at E12 [11]. However, stimulation of auditory neurons is also observed at E5 [1], when no expression of NT-
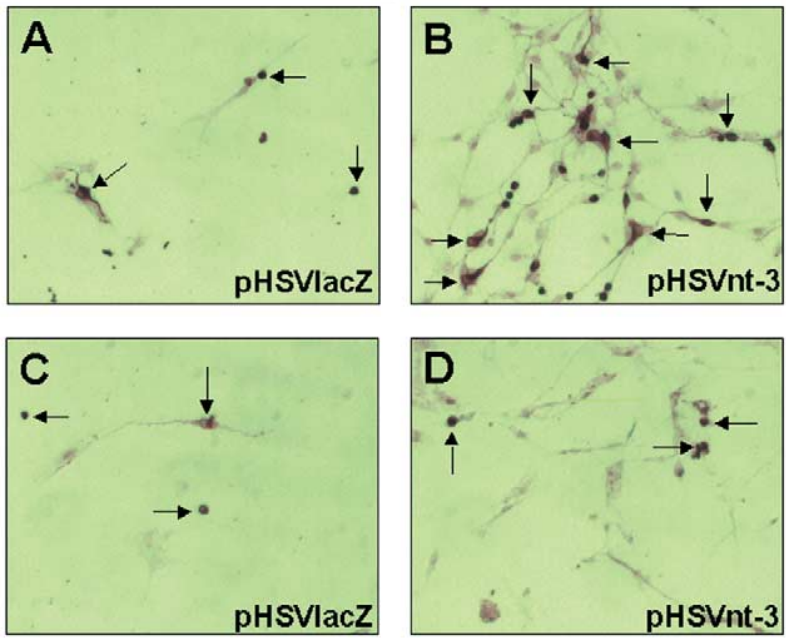

Fig. 2. Images of cochlear neuron cultures isolated at day $8(A, B)$ or day $14(C, D)$ of chicken development which have been transduced with pHSVlacZ $(A, C)$ or pHSVnt-3 $(B, D)$. After $48 \mathrm{~h}$ in culture, cells were fixed and stained with a tubulin antibody to visualize neurons. Cultures prepared at embryonic day 8 and infected with pHSVnt-3 show increased neuronal survival compared with control cultures infected with pHSVlacZ. At day 14 of development, similar amounts of neurons are observed in both pHSVlacZ- and pHSVnt-3-transduced cultures. Neuronal cultures were infected with $1.65 \times 10^{4} \mathrm{ivu} / \mathrm{ml}$ of $\mathrm{pHSVnt}-3$ and $3.1 \times 10^{4} \mathrm{ivu} / \mathrm{ml}$ of $\mathrm{pHSVlacZ}$, respectively. Arrows point out neurons stained with the tubulin antibody. Scale bar, $100 \mu \mathrm{m}$.

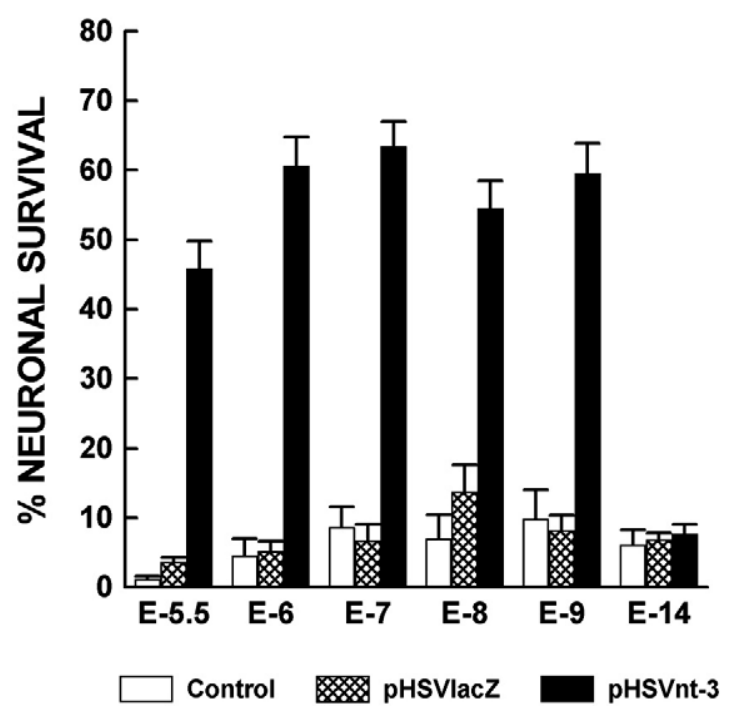

Fig. 3. Temporal pattern of neurotrophic effects of virally transferred NT-3 on chicken auditory neurons. Bar charts show the survival of mock-, pHSVlacZ- and pHSVnt-3-infected neurons isolated at the embryonic day of development $(E)$ indicated, after $48 \mathrm{~h}$. The results are expressed as the means \pm SD of three separate determinations for each experimental condition. The data shown demonstrate a clear survival-promoting effect of pHSV nt-3 between E5.5 and E9, whereas neurons do not respond at E14. Values from cultures infected with pHSV nt-3 are statistically different from values for $\mathrm{pHSVlacZ}$ and the uninfected control $(P<0.001, t$-test $)$ at E5.5-E9.

3 has been detected [11]. The most likely explanation for this discrepancy is the presence of high-affinity receptors for NT3 , encoded by TrkC, which are expressed during these stages [2]. TrkC expression thus precedes the expression of NT-3 in vivo. Additionally, TrkC is also maintained during later embryogenesis and the post-hatch period, when NT-3 is possibly supplied by central sources [2]. In summary and as suggested earlier, NT-3 appears to play only a transitory role during chicken development $[2,11]$. This situation is strikingly different in mammals, where NT-3 is expressed throughout development and is the only neurotrophin present at adult stages $[18,19]$. In the adult cochlea, NT-3 is expressed in inner hair cells, which are innervated by type I sensory neurons which comprise the vast majority of cochlear neurons in mice. The importance of this neurotrophin during development is convincingly demonstrated by the loss of more than $80 \%$ of cochlear sensory neurons in mice lacking NT-3 [5]. In contrast and as expected, lack of signaling via other neurotrophin members only produces minor or no defects to auditory system development [5]. NT-3 thus may be considered the most promising candidate to provide neurotrophic support for mammalian auditory sensory neurons, which are at risk of undergoing neurodegenerative processes. Indeed, in vitro models for neurodegeneration provided evidence that NT-3 is the most efficient neurotrophin for survival of maturing auditory neurons [15]. Moreover, neuronal degeneration caused by ototoxins was largely prevented by NT-3 in vitro, although BDNF and NT- 
4 were shown to act with similar efficacy [20]. Most importantly, upon infusion via osmotic pumps, NT-3 has consistently protected cochlear neurons from noise- and aminoglycoside-induced ototoxicity $[3,4,13,16]$. In this context, NT-3 has additionally been shown to participate in the protection of hair cells [3]. BDNF has also been reported to prevent loss of auditory neurons in vivo, although with less efficiency than NT-3 [16]. The fact that NT-3 may provide a potent protection of auditory sensory neurons in vivo prompted us to develop a viral vector expressing this neurotrophin. Previous studies have described HSV-1-based amplicon vectors with similar characteristics expressing BDNF, which were shown to stimulate neuronal survival and prevent degeneration of auditory neurons [7,8,17]. Compared with other viral vectors, like for instance adenovirus, HSV-1-based vectors are unique in their capacity to infect neurons very efficiently and harbor the potential to provide a life-long supply of the gene product expressed by the vector, thereby creating an endogenous source and eliminating the need to use osmotic pumps. Our results demonstrate the HSV-1-mediated expression of biologically active NT-3 in auditory sensory neurons, which efficiently stimulates their survival in vitro. Future experiments will now be directed to explore the neuroprotective capacity of this vector in the inner ear and other NT-3-dependent peripheral sensory systems in vivo.

The results of this study highlight the unique importance of NT-3 for the formation of the peripheral nervous system innervating the inner ear and the survival of auditory sensory neurons. The efficient transfer of this neurotrophin into these neurons via a HSV-1-based amplicon vector underlines the capacity of this system to permanently supply the inner ear with neurotrophic molecules. The future application of this vector will include the introduction of NT-3 into auditory sensory neurons in vivo which may offer a decisive key step to prevent their degeneration and thus to ameliorate or avoid hearing impairment.

The authors would like to thank Fernando Giraldez for his generous support of this project. This research was supported by DGCYT and the Junta of Castilla y León. Recombinant NT-3 was provided by Regeneron.

[1] Avila, M.A., Varela-Nieto, I., Romero, G., Mato, J.M., Giraldez, F., Van de Water, T. and Represa, J., Brain-derived neurotrophic factor and neurotrophin-3 support survival and neuritogenesis response of developing cochleovestibular ganglion neurons, Dev. Biol., 159 (1993) 266-275.

[2] Cochran, S.L., Stone, J.S., Bermingham-McDonogh, O., Akers, S.R., Lefcort, F. and Rubel, E.W., Ontogenetic expression of Trk neurotrophin receptors in the chick auditory system, J. Comp. Neurol., 413 (1999) 217-288.

[3] Duan, M., Agerman, K., Ernfors, P. and Canlon, B., Complementary roles of neurotrophin 3 and a $N$-methyl-D-aspartate antagonist in the protection of noise and aminoglycosideinduced ototoxicity, Proc. Natl. Acad. Sci. USA, 97 (2000) 7597-7602.

[4] Ernfors, P., Duan, M.L., ElShamy, W.M. and Canlon, B.,
Protection of auditory neurons from aminoglycoside toxicity by neurotrophin-3, Nat. Med., 2 (1996) 463-467.

[5] Fritzsch, B., Silos-Santiago, I., Bianchi, L.M. and Farinas, I., The role of neurotrophic factors in regulating the development of inner ear innervation, Trends Neurosci., 20 (1997) 159-164.

[6] Gao, W.-Q., Therapeutic potential of neurotrophins for treatment of hearing loss, Mol. Neurobiol., 17 (1998) 17-31.

[7] Garrido, J.J., Alonso, M.T., Lim, F., Carnicero, E., Giraldez, F. and Schimmang, T., Defining responsiveness of avian cochlear neurons to brain-derived neurotrophic factor and nerve growth factor by HSV-1-mediated gene transfer, J. Neurochem., 70 (1998) 2336-2346.

[8] Geschwind, M.D., Hartnick, C.J., Liu, W., Amat, J., Van de Water, T. and Federoff, H.J., Defective HSV-1 vector expressing BDNF in auditory ganglia elicits neurite outgrowth: model for treatment of neuron loss following cochlear degeneration, Hum. Gene Ther., 7 (1996) 173-182.

[9] Lim, F., Hartley, D., Starr, P., Song, S., Yu, L., Wang, Y. and Geller, A.I., Generation of high-titer defective HSV-1 vectors using an IE2 deletion mutant and quantitative study of expression in cultured cortical cells, Biotechniques, 20 (1996) 460-469.

[10] Pirvola, U., Arumäe, U., Moshynakov, M., Palgi, J., Saarma, M. and Ylikoski, J., Coordinated expression and function of neurotrophins and their receptors in the rat inner ear during target innervation, Hear. Res., 75 (1994) 131-144.

[11] Pirvola, U., Hallböök, F., Xing-Qun, L., Vikkala, J., Saarma, M. and Ylikoski, J., Expression of neurotrophins and Trk receptors in the developing, adult and regenerating avian cochlea, J. Neurobiol., 33 (1997) 1019-1033.

[12] Schecterson, L. and Bothwell, M., Neurotrophin and neurotrophin receptor mRNA expression in developing ear, Hear. Res., 73 (1994) 92-100.

[13] Shoji, F., Miller, A.L., Mitchell, A., Yamasoba, T., Altschuler, R.A. and Miller, J.M., Differential protective effects of neurotrophins in the attenuation of noise-induced hair cell loss, Hear. Res., 146 (2000) 134-142.

[14] Simonato, M., Manservigi, R., Marconi, P. and Glorioso, J., Gene transfer into neurones for the molecular analysis of behaviour: focus on herpes simplex vectors, Trends Neurosci., 23 (2000) 183-190.

[15] Staecker, H., Galinovic-Schwartz, V., Liu, W., Lefebvre, P., Kopke, R., Malgrange, B., Moonen, G. and Van de Water, T.R., The role of neurotrophins in maturation and maintenance of postnatal auditory innervation, Am. J. Otol., 17 (1996) 486-492.

[16] Staecker, H., Kopke, R., Malgrange, B., Lefebvre, P. and Van de Water, T.R., NT-3 and/or BDNF therapy prevents loss of auditory neurons following loss of hair cells, NeuroReport, 7 (1996) 889-894.

[17] Staecker, H., Gabaizadeh, R., Federoff, H. and Van de Water, T., Brain-derived neurotrophic factor gene therapy prevents spiral ganglion degeneration after hair cell loss, Otolaryngol. Head Neck Surg., 19 (1998) 7-13.

[18] Wheeler, E., Bothwell, M., Schecterson, L.C. and von Bartheld, C.S., Expression of BDNF and NT-3 mRNA in hair cells of the organ of Corti: quantitative analysis in developing rats, Hear. Res., 73 (1994) 46-56.

[19] Ylikoski, J., Pirvola, U., Moshynakov, M., Palgi, J., Arumäe, $U$. and Saarma, M., Expression patterns of neurotrophin and their receptor mRNAs in the rat inner ear, Hear. Res., 56 (1993) 69-78.

[20] Zheng, J.L. and Gao, W.Q., Differential damage to auditory neurons and hair cells by ototoxins and neuroprotection by specific neurotrophins in rat cochlear organotypic cultures, Eur. J. Neurosci., 8 (1996) 1897-1905. 